\title{
CHALLENGES IN THE DIAGNOSIS OF PEDIATRIC PNEUMONIA
}

\author{
Ligia Georgeta Stanescu ${ }^{1}$, Catalina Bica ${ }^{1}$, Cristian Gheonea ${ }^{1}$, Radu Diaconu ${ }^{1}$, \\ Ramona Nedelcuta', Liliana Anghelina', Loredana Selaru' ${ }^{2}$, Andreea Pistolea ${ }^{2}$ \\ I"Filantropia" Pediatric Clinic of Municipal Hospital, \\ University of Medicine and Pharmacy. Craiova \\ 2 "Filantropia” Pediatric Clinic of Municipal Hospital, Craiova
}

\begin{abstract}
Pneumonia is a form of acute respiratory infection that affects the lungs and it is the leading cause of illness and death in children worldwide. Pneumonia can occur at any age, but it is more common in younger children. The etiology of pneumonia is often difficult to identify and the diagnostic criteria is based on corroborating epidemiological, clinical, laboratory and radiologic investigations. The authors present three cases diagnosed with pneumonia at different ages, underlying the clinical, imagistic and evolutive particularities.
\end{abstract}

Keywords: pneumonia, children, age, outcome

\section{INTRODUCTION}

Pneumonia is the leading cause of illness and death in children worldwide, with an estimated global annual incidence of 150 to 156 million cases in children less than 5 years old, of whom approximately 11 to 20 million need hospitalization and 1.1 million die of this condition (1). The United Nations Children's Fund (UNICEF) estimates even a bigger number, of 3 million children deceased of pneumonia worldwide each year, with the particularity that it occurs almost exclusively in children with underlying conditions, such as chronic lung disease of prematurity, congenital heart disease and immunosuppression. Although most fatalities take place in the developing countries, pneumonia remains a significant cause of morbidity even in industrialized nations (2). Overall, pneumonia accounts for $18 \%$ of the total number of deaths in children under 5 years old worldwide, more than tuberculosis, AIDS and malaria combined (1).

Pneumonia can occur at any age, although it is more common in younger children and it accounts for $13 \%$ of all infectious illnesses in infants younger than 2 years old . Respiratory viruses are responsible for the most cases of community-acquired pneumonia among hospitalized children. (3)
Pediatric pneumonia remains a diagnostic challenge in countries with limited resources. In many cases, the diagnosis of pneumonia in children is based on clinical criteria and a good physical examination that can usually detect typical symptoms of pneumonia and localize the infection. The role of imaging (chest radiographs, in particularly) in confirming the diagnosis of pneumonia is important when the physical examination is inconclusive or difficult. These cases are usually meet in younger children and infants, where signs and symptoms may be non-specific or subtle $(4,5)$.

The authors present three cases diagnosed with pneumonia at different ages, underlying the clinical, imagistic and evolutional particularities.

\section{Case one}

Patient G.R., 7 months old, female, from rural area is admitted for tachypnea, wheezing, cough and nasal obstruction. The illness begun a month before with signs of acute rhinopharyngitis, followed by shortness of breath needing hospitalization at the local hospital. After discharge, the patient's evolution was unsatisfying, with moderate respiratory syndrome, which was the cause for admittance in our clinic. Heredocolateral and personal physiological antecedents were irrelevant. 
The clinical exam revealed a patient with altered status, no fever, pallor, 7,800 grams, cough, nasal discharge, $42 \mathrm{resp} . / \mathrm{min}$, wheeze, retractions at intercostal and subcostal sites, prolonged expiration, pulmonary crackles (bronchial and subcrepitant rales) $100 \mathrm{~b} / \mathrm{min}$, liver at the costal border, digestive and urinary system without alteration. The laboratory investigations revealed moderate elevated inflammatory reactants $(45 / 86 \mathrm{~mm}$ ESR, C reactive protein under $6 \mathrm{mg} / \mathrm{dl}$ ) normal leucocytes level, with predominant lymphocytes $(67 \%)$. The chest radiograph revealed right perihilar opacity (Fig. 1). Ear, nose and throat (ENT) examination showed acute rhinopharyngitis. The patient received treatment with antibiotics (parenteral cefuroxime five days, followed by ceftriaxone), inhaled bronchodilatator and steroid therapy, oxygen therapy when needed and symptomatic therapy.

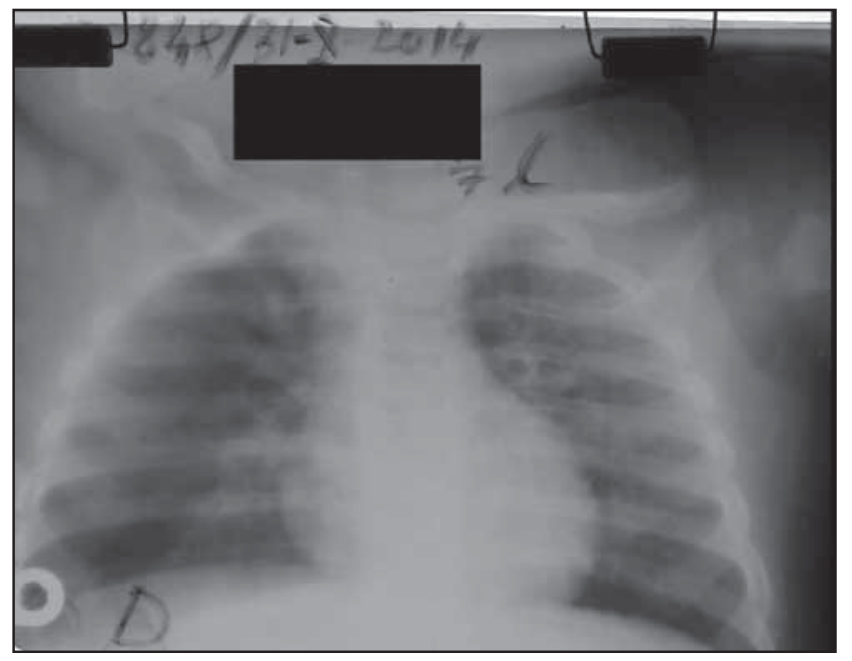

FIGURE 1. Chest radiograph of case no. 1 showing right perihilar opacity

The evolution was unsatisfying, with periods of exacerbation of the ventilator syndrome with increased breath rhythm (58-60 r/min) and ventricular rate $(120 / \mathrm{min})$, hepatomegaly $(1,5 \mathrm{~cm}$ below costal border) that needed the introduction of diuretic therapy. Oxygen saturation on pulse oximetry was $90 \%$. In the fourth day of admittance, the patient presented fever, vomiting, loss of appetite and diarrhea and received endovenous perfusion and pathogenic therapy. Under treatment, the digestive episode reunitted, but the ventilator syndrome was still present. Pharyngeal, blood and stool cultures were negative. Another blood test was repeated with comparable result, normal leucocytes level $(8,500 / \mathrm{mmc})$ and a slight elevation of the neutrophils percentage (50\%). ESR maintained a similar level $(32 / 56 \mathrm{~mm})$.

Taking into consideration the clinical progress without improvement under therapy, the X-ray as- pect that suggested hilar lymphadenopathy and the poor living status, the patient was transferred to the Pneumophtisiology Clinic of the Infectious Disease "Victor Babes" of Craiova under the suspicion of primary pulmonary tuberculosis. The diagnostic was "Bronchopneumonia. Hilar lymphadenopathy. Rhinopharyngitis". Further tests were done; Chest $\mathrm{X}$-ray was repeated and it identified a development of the right pulmonary opacity in the form of a paratracheal broad band. Tuberculin skin test was negative and the early-morning gastric aspirate did not identify the Koch bacillus. Because specific etiology (Koch bacillus) was not acknowledged, antibiotic therapy was continued for two more weeks, alongside pathogenic therapy until the resolution of respiratory symptoms. The outcome was positive, without relapses and had a total resolution of the pulmonary opacity.

\section{Case 2}

P.M., 4 years old, female, from rural area is admitted for irittative cough, nasal obstruction and dysphagia. The disease began a week before, with unspecific signs, loss of appetite, fever and rare dry cough. She received treatment at the local hospital with intravenous antibiotics and symptomatic therapy with no positive results, therefore she was transferred to the Pneumophtisiology Clinic. Physical examination revealed a modified status, fever, dark circles around the eyes, productive cough, elevated respiratory frequency $44 / \mathrm{min}$, pulmonary submatity at percussion, fine subcrepitant rales in the third inferior area of the right lung and hyperemic pharynx. Blood tests showed normal hemoglobin level (12.4 g/dl) moderate leucocytoses $(12,000 / \mathrm{mmc})$ with predominance of lymphocytes $59 \%$. ESR level was $15 / 35 \mathrm{~mm}$. Other laboratory investigations were inconclusive. ENT exam revealed acute rhinopharyngitis and acute medium otitis. Chest X-ray showed homogenous opacity in the third inferior area of the right lung (Fig. 2). Tuberculin skin test was negative. The diagnosis was "Right inferior lobe acute pneumonia. Acute rhinopharyngitis. Acute medium otitis". Treatment began with intravenous ceftriaxone, 8 days and symptomatic therapy followed by oral antibiotherapy (amoxicilin-clavulanate, 7 days). The outcome was favorable with resolution of the symptoms and the pulmonary opacity.

\section{Case 3}

M.A.C., 16 years old, male, from urban area was admitted for productive cough, fever, shiver, 


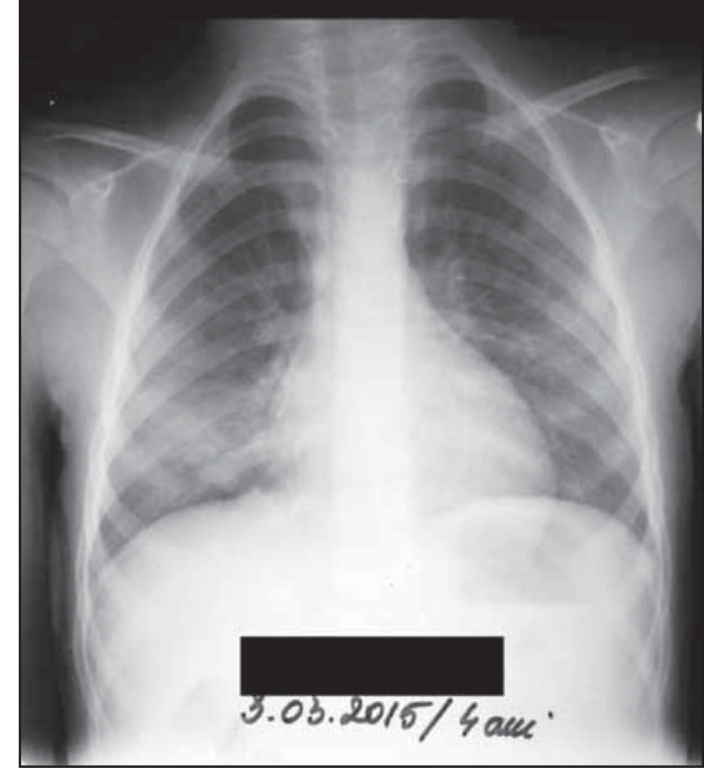

FIGURE 2. Chest $X$-ray of case no. 2

dyspnea, thoracic pain, anorexia. Clavulate-amoxicillin (1 $\mathrm{g}$ two times a day) was given in ambulatory therapy with no positive result. Personal pathological and heredocolateral antecedents were insignificant for current illness. Clinical examination revealed altered status, fever, dark circles around the eyes, hyperemia of the pharynx, elevated respiratory frequency (44/min), matity at percussion of the left base of the lung, fine crepitant rales in the same region. Cardiac, digestive and renal systems were without modifications. Laboratory findings included normal hemoglobin level (14,3 g/dl) and normal leucocytes level $(7910 / \mathrm{mmc})$ with predominance of the neutrophils $(65 \%)$ on the leucocitary formula, moderate elevated ESR level $(35 / 70 \mathrm{~mm})$. Also, the transaminases levels were elevated (ALT 141, 3UI/1; AST 85,4UI/1), probably due to antibiotics consumption. ENT examination showed posterior rhinorrhea and X-ray investigation revealed heterogeneous diffuse opacity in the $2 / 3$ of the inferior left lung with pleural effusion and scizuritis (Fig. 3). The diagnostic was "Left lobe acute pneumonia. Pleuritis. Scizuritis. Hepatocytolysis". The patient began treatment with intravenous ampicillin for 5 days followed by oral penicillin ( $1 \mathrm{~g}$, three times per day, 10 days) systemic corticoid therapy to relieve the ventilatory syndrome, liver protection and symptomatic therapy. The outcome was favorable under treatment, with the resolution of pulmonary modifications.

\section{DISCUSSION}

Pneumonia is a form of acute respiratory infection that affects the lungs. According to the World

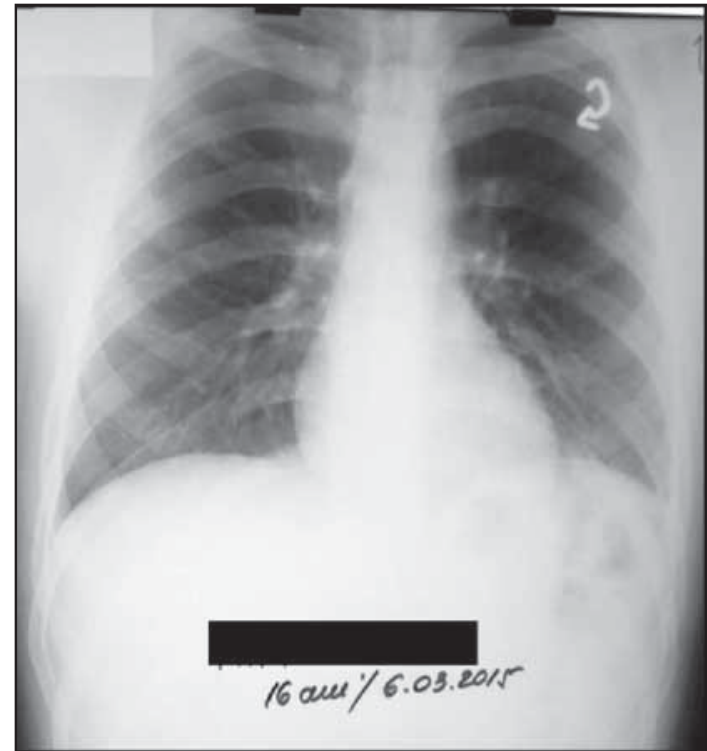

FIGURE 3. Chest $X$-ray of case no. 3

Health Organisation (WHO) pneumonia is the single largest infectious cause of death in children worldwide, killing over 922,000 children under the age of 5 in 2015, accounting for $15 \%$ of all deaths of children under five years old (6). Pneumonia can occur at any age, although it is more common in younger children; it accounts for $13 \%$ of all infectious illnesses in infants younger than 2 years. Pneumonia is caused by a number of infectious agents, including viruses, bacteria and fungi. The most common cause of bacterial infection in children is Streptococcus pneumoniae, followed by Haemophilus influenzae type $b$ (Hib). Among viral infection causing pneumonia, respiratory syncitial virus is the most frequent, being responsible for most cases of community-acquired pneumonia among hospitalized children. Altogether, respiratory viruses place a great burden, especially in younger children in terms of high rates infection, bacterial complications and hospitalizations. In developing countries, some viral infections are even associated with mortality. New findings may redirect clinical decisions and prescriptions, according to experts $(3,4,6,7)$.

The viruses and bacteria that are commonly found in a child's nose or throat can infect the lungs if they are inhaled. They may also spread via airborne droplets from a cough or sneeze. In addition, pneumonia may spread through blood, during and shortly after birth $(4,6)$.

The presenting features of viral and bacterial pneumonia are similar. However, the symptoms of viral pneumonia may be more numerous than the symptoms of bacterial pneumonia. Tachypnea has 
the biggest predictive value for the diagnosis of pneumonia, especially in children less than 3 years. Cough is the most common symptom of pneumonia in infants, along with tachypnea, chest retractions and hypoxemia. These may be accompanied by congestion, fever, irritability, and decreased feeding, especially in very severely ill infants. Wheezing is more common in viral infections. Adolescents experience symptoms similar to younger children's. They may have other constitutional symptoms, such as headache, pleuritic chest pain and vague abdominal pain. Vomiting, diarrhea, pharyngitis, and otalgia/otitis are also common in this age group. Mycoplasma pneumoniae is the most frequent cause of pneumonia among older children and adolescents $(2,4,8,9)$.

The cases we have presented started at different ages and have had an unfavorable, prolonged evolution. X-rays patterns were suggestive for primary tuberculosis and admission in a Pneumophtisiology hospital was required in order to exclude this diagnosis. The onset was different, subtle and preceded by catharale rhinopharyngitis signs in the smallest children, while in the third case, the adolescent had a sudden onset. These characteristics of the onset are also found in other medical papers.

The respiratory distress syndrome was moderate in all three cases, but with a more severe and prolonged prospective in case of the infant. The hemodynamics changes were as a result of hypoxia and we highlight elevated ventricular rate, lowering of the liver inferior border, fine subcrepitates rales that resolved after pathogenic therapy, without cardiac impairment. The toxic-infectious syndrome was present in all cases. The infant had a more severe evolution, probably due to its reduced transplacental immunoglobin level and decreased immunoreactive capacity. Also, he associated an acute diarrhea, that complicated the bronchopneumonia. Most healthy children can fight the infection with their natural defences systems. Children whose immune systems are compromised by malnutrition or preexisting illnesses (HIV infection, measles) are at higher risk of developing pneumonia, especially infants who are not exclusively breastfed. None of our cases had preexisting illness. Also, environmental factors like parental smoking, living in crowded houses and air pollution increase a child's susceptibility to pneumonia $(6,10)$. From our cases, the first patient had a poor living status, but they did not present any other environmental associated factors.
Radiological findings in the studied cases identified pseudo-lobar bronchopneumonia aspects without obstructive complications (atelectasis) in the two smallest children and a pneumonia pattern in $2 / 3$ of the inferior left pulmonary lobe, in the third case. Further more, all cases have had blood results that did not described substantial bacterial infections. The first case showed a turning in the leucocitary formula from lymphocytosis to neutrophilia. Viral testing was not performed, but we suspect that it could have had a positive result.

There are numerous studies that confirm the role of viruses in pediatric pneumonia. It is cited a study from 2015 that enrolled 2,638 children diagnosed with pneumonia. Researchers found that although $89 \%$ of all patients enrolled had radiographic evidence of pneumonia, $66 \%$ were positive for one or more viruses and only $8 \%$ had bacterial infection. $7 \%$ had both viral and bacterial pathogens. The majority of children were younger than 4 years old $(70 \%), 45 \%$ of them being under 2 years old. Also, the study shows that the respiratory syncitial virus was the most common found (28\%) from all samples and in patients under 2 years old. The second most common virus overall was the rhinovirus. Regarding the age under 5 years old, two other viruses were identified, adenovirus and metapneumovirus. In patients over 5 years old, Mycoplasma pneumonie was present in 19\% of cases (3). The interaction between viruses and bacteria is probably much more common and clinically significant than previously understood; one in four studied children had multiple pathogens, according to other studies $(7,11)$. It is known that viruses usually initiate the cascade of events that ultimately leads to bacterial infection. In developed countries, the low prevalence of bacterial infection in pneumonia probably reflects the effectiveness of bacterial conjugate vaccines. Also, the diagnostic tests are relatively insensitive, hence the necessity of a faster, less-expensive test for accurately diagnose the cause of pneumonia.

Relating this study with our cases, we can suppose that viruses could have been the predominant etiology or that it appeard an interaction between viruses and bacteria, that shaped the course of the disease. In addition, none of our patients received pneumococcal conjugate vaccination (PCV).

An attempt has been made to elaborate a diagnostic score, in order to identify bacterial pneumonias, even without positive cultures. This score includes clinical, laboratory and radiological findings easy to recognize, as seen in Tables 1 and 2 (4). 
TABLE 1. Radiological pediatric score for the diagnosis of pneumonia (4)

\begin{tabular}{|l|c|}
\hline 1. Infiltrates (condensation) & +2 \\
\hline Well defined: lobar, segmental, lobular & +1 \\
\hline $\begin{array}{l}\text { Patchy, irregular densities hard to defined, (alveolar, } \\
\text { lobular) }\end{array}$ & -1 \\
\hline Peribronchial pattern & +1 \\
\hline 2. Localization & +1 \\
\hline One lobe & -1 \\
\hline Multiple lobules or both pulmons & \\
\hline Multiple areas, predominant perihilar & +1 \\
\hline 3. Pleural space & +2 \\
\hline Minimal pleural effusion (only costo-diafragmatic angle) & \\
\hline Pleural effusion & +1 \\
\hline 4. Abscesses, pneumatoceles & +2 \\
\hline Ambiguity & \\
\hline Well defined & -1 \\
\hline 5. Atelectasis & -1 \\
\hline Subsegmental & 0 \\
\hline Lobar (right, superior or middle lobe) &
\end{tabular}

Results can be interpreted like this: bacterial infection $\geq 1$, viral pneumonia 1.5-3; pneumococcal pneumonia 4.4; staphylococcal pneumonia 6.5 ; Haemophilus influenzae pneumonia 1.5.

TABLE 2. Diagnostic score for bacterial pneumonias (4)

\begin{tabular}{|l|c|}
\hline Radiological score - see Table 1 & \\
\hline Leucocytoses $(>20,000 / \mathrm{mmc})$ & +1 \\
\hline $\begin{array}{l}\text { Neutrophils, in absolute number } \\
(>10,000 / \mathrm{mmc})\end{array}$ & +1 \\
\hline Left deviation on leucocitary formula & +1 \\
\hline Fever $\left(>39^{\circ} \mathrm{C}\right)$ & +1 \\
\hline C reactive protein $(>20 \mathrm{mg} / \mathrm{dl})$ & +1 \\
\hline
\end{tabular}

If we apply the radiological score in our cases, the results are not relevant (score 2 and 3 for the three cases described that did not help us distinguish between viral or bacterial pneumonia). Regarding the $\mathrm{X}$-ray investigation, it is known that the chest radiograph is the primary imagistic study used to confirm the diagnosis of pneumonia. Numerous radiographic patterns are consistent with pneumonia, but also a multitude of other pathologic entities. When considering pneumonia, it is good to take into consideration that any image reflects a condition only at that instant at which the study was performed. Because pneumonia is a dynamic illness, initially suggestive images may require reassessment based on the subsequent clinical course $(4,5)$. All our patients received follow-up chest Xrays that confirmed the clinical status and in the end the resolution of the disease. Furthermore, it is known that X-ray imaging presents frequently a number of non-specific patterns and it is demon- strated that is only $42-73 \%$ accurate in predicting the etiology of pneumonia (5). Wubbel et al established in a study on community-acquired pneumonia made on 168 children, who were independently evaluated by 2 radiologists, that they were unable to distinguish whether the agent involved was bacterial, viral or unidentified (12). The obvious conclusion is that for the pneumonia diagnose, is must be considered the imaging, clinical presentation and laboratory findings to help physicians establish the etiologic agent $(4,12)$.

Indications for chest radiography depend on the age of the patient. Infants and toddlers who present fever with signs of respiratory distress, like tachypnea, nasal flaring, retractions, rales, decreased breath sounds have the indication for X-ray. On the other side, in older children and adolescents, chest $\mathrm{X}$-ray may not be necessary if the clinical presentation is evident for the diagnosis of pneumonia $(4,5)$. Another aspect worth taking into consideration is that of lobar infiltrates usually seen in bacterial pneumonia. Several studies have found that the pattern of radiologic features cannot accurate distinguish a bacterial from a viral infection $(13,14)$. It is cited a study which demonstrated that even if a large number of cases have had radiologic findings of pneumonia, the vast majority had pneumonia caused by a virus (3). In contrast, a Finnish study concluded that alveolar (equivalent to lobar) infiltrate is an insensitive, but reasonable specific indicator of bacterial infection. Thus, a lobar infiltrate can be seen with viral infections, with bacterial infections or with another type of entities (like foreign body aspiration, which wasn't the case in this study) (15).

It exists recent interest in developing new tools needed to increase the accuracy of pneumonia diagnosis while decreasing exposure to ionizing radiation. Advances in the ultrasound technology have made lung ultrasound (LUS) an attractive option for the diagnosis of pneumonia $(1,2)$. A metaanalysis comparing the use of LUS against a reference standard for the diagnosis of pneumonia (clinical diagnosis, chest radiograph $+/$ - blood results) has found that the sensitivity of LUS was similar when clinical and chest radiograph were used to define childhood pneumonia (1). Similar studies have found comparable results when both children and adults were included. Regarding the quality of LUS examination results vary depending on body mass and thorax size. In children and neonates, the smaller thoracic diameter and lung volume permit a better visualization with LUS. Moreover, LUS is safe, portable, inexpensive and relatively easy to teach general pediatricians $(1,16)$. 
Among cases we described, a special consideration is neded by case no. 1, where the first radiograph was suggestive for primary pulmonary tuberculosis (hilar adenopathy). A history of TB exposure should be obtained in every patient who presents with signs and symptoms of pneumonia. Children with TB usually do not present with symptoms until 1-6 months after primary infection. Chest radiograph findings in children with tuberculosis may include hilar lymphadenopathy, atelectasis, consolidation of a segment or lobe (usually, right upper lobe) pleural effusion or milliary disease. Case no. 2 also presented a consolidation, but of the right inferior lobe and the third case had a pleuritic reaction. All three cases had negative tuberculin skin test. The attempt to isolate tuberculous bacilli from early-morning gastric aspirate remained negative in all cases.

There are many studies that show the effectiveness of pneumococcal conjugate vaccine (PCV) against pneumonia and other noninvasive conditions (like sinusitis), as shown by Lindstrand et al in a 2014 study published in Pediatrics. The Swedish authors proved a significantly reduction of sinusitis hospitalization after PCV7 and PCV13 vaccination in children younger than 5 years old. Also, there was a reduction in hospitalization rates for pneumonia in the same age group (17). Other studies showed even a more decreased incidence of various pneumococcal illnesses, until almost disappearance in sinusitis, but with an increase in $S$. aureus as cited by Pēna et al, in a study from United States or Fitzwater et al who showed a reduction of $65 \%$ of pneumonia hospitalization. $(18,19)$ In Europe, children younger than 2 years constitute the population most at risk for pneumococcal infection, with the rates decreasing with age. The overall incidence of the invasive disease is estimated to be lower in Europe (14 per 100,000 persons in Germany vs 35.8 per 100,000 persons in England vs 45.3 per 100,000 persons in Finland vs 90 per 100,000 persons in Spain) than in the United States
(235 per 100,000 persons) (2). These findings are convincing for the use of PCV and we consider of most importance that PCV be included in Romania's national vaccination schedule. Otherwise, the cost of the vaccine could be an impairment for most of the parents and the effect of the PCV is most comprehensive when a larger population at risk is vaccinated.

In all three cases, the etiology could not be identified due to initial antibiotics use, therefore the diagnosis of pneumonia was made on clinical, radiological and laboratory exams (positive acute phase reactants and negative tuberculin skin tests that suggested bacterial infection, by exclusion). According to WHO, pneumonia should be treated with antibiotics and hospitalization should be recommended only for severe cases of pneumonia. The antibiotic of choice is amoxicillin and most cases of pneumonia require oral antibiotics, which could be often prescribed at a health centre. Cases that can be diagnosed and treated with inexpensive oral antibiotics by trained community health workers have a less impact on the health system $(6,8)$.

\section{CONCLUSIONS}

In conclusion, all cases we presented have had a prolonged evolution. The etiology was difficult to determine. The interaction between viruses and bacteria is much more common and clinically significant than previously understood by practicians. Diagnostic tests are relatively insensitive, hence the necessity of a faster, less-expansive test for accurately diagnose the cause of pneumonia. New tools for diagnosis of pneumonia have been found that can improve the accuracy of pneumonia diagnosis. Preventing pneumonia in children is an essential component of a strategy to reduce children mortality and it should be the first step for improving the health care in pediatric patients. This may be possible by the introduction, in Romania, of a national program $\mathrm{PCV}$ vaccination.

\section{REFERENCES}

1. Pereda M., Chavez M., Hooper-Miele C. et al. Lung ultrasound for the diagnosis of pneumonia in children: a meta-analysis, Pediatrics 2015; vol.135:714-722.

2. Bennet J.N., Domachowski J., Steele R.W. Pediatric Pneumonia, emedicine.medscape.com/article/967822 updated May $3^{\text {rd }}, 2016$.

3. Laidman J. Viral infection most common cause of pneumonia in children, N Engl J Med 2015; 372:835-845.

4. Ciofu E., Ciofu C. Esențialul în Pediatrie. Ed. Medicală Amaltea, Bucureşti 2002.

5. Bennett N. Imaging in Pediatric Pneumonia: Introduction, Chest Radiography, Chest CT Scanning, emedicine.medscape.com/ article/19269880, updated Nov 22, 2015

6. Pneumonia. www.who.int/mediacentre/facsheets/fs331/en updated November 2015

7. Heikkinen T. Respiratory viruses and children. Abstract in $\mathrm{J}$ Infect 2016, May 10, epub ahead of print

8. Nanulescu M. Pneumonia Comunitară în Protocoale de diagnostic şl tratament în Pediatrie sub coord. Prof. Dr. Nanulescu Mircea, Ed. Medicală Amaltea, Bucureşti 2012. 
9. Nieves Prado A., Perloff S. Pneumococcal Infections, emedicine. medscape.com/article/225811, updated Jan 22, 2016

10. Souza L.S., Nascimento L.F. Air pollutants and hospital admission due to pneumonia in children: a time series analysis. Rev Assoc Med Bras 2016; 622(2):151-156.

11. Lee C.H., Won Y.K., Roh E.J. et al. A nationwide study of children and adolescents with pneumonia who visited Emergency Department in South Korea in 2012, Korean J Pediatr 2016; 59(3):132/138.

12. Wubbel I., Ahmed A., Trujillo M. et al. Etiology and treatment of community-acquired pneumonia in ambulatory children. Pediatr Infect Dis J, 1999; 18(2):98-104.

13. Haney P.J., Bohlman M., Sun C.C. Radiographic findings in neonatal pneumonia, AJR Am J Roentgenol, 1984; 143(1):23-26.

14. Courtoy I., Lande A.E., Turner R.B. Accuracy of radiographic differentiation of bacterial from non-bacterial pneumonia, Clin Pediatr (Phila) 1989; 28(6):261-264.
15. Korppi M., Kiekara O., Heiskanen-Kosma T. et al. Comparison of radiological findings and microbial aetiology of childhood pneumonia, Acta Pediatr 1993; 82(4):360-363.

16. Reissig A., Copetti R. Lung ultrasound in community-acuired pneumonia and in interstitial lung diseases, Respiration, 2014; 87(3):179-189.

17. Lindstrand A., Rutger B., Galanis et al. Sinusitis and pneumonia hospitalization after introduction of pneumococcal conjugate vaccine, Pediatrics 2014; 134(6):1-9.

18. Peña M.T., Preciado D., Orestes M. et al. Orbital complications of acute sinusitis: changes in the post-pneumococcal vaccine era. JAMA Otolaryngol Head Neck Surg. 2013; 139(3):223-227.

19. Fitzwater S.P., Chandran A., Santosham M. et al. The worldwide impact of the seven-valent pneumococcal conjugate vaccine Pediatr Infect Dis J. 2012; 31(5):501-508. 